\title{
Synthesis of some new 5-amino-3-(substituted-amino)-6-(fluoro/ nitro)aryl-1,2,4-triazine derivatives as lamotrigine analogs and their evaluation in vitro as antibacterial agents
}

\author{
Abdulrahman S. Alharbi ${ }^{1, *}$ and Nawaa A. Alshammari ${ }^{1,2}$ \\ ${ }^{1}$ Department of Chemistry, Faculty of Science, King Abdul Aziz University, Jeddah, Saudi Arabia \\ ${ }^{2}$ Department of Chemistry, Faculty of Science, Northern Borders University, Rafha, Saudi Arabia
}

\begin{abstract}
Some new fluorine-substituted 3,5-disubstituted amino-1,2,4-triazines have been obtained from arylamination of 2,2,2-trifluoro- $N$-[2-(5-hydroxy-3-thioxo-2,3-dihydro-1,2,4-triazin-6-yl)-4-nitrophenyl] acetamide followed by ammonolysis to produce $N$-(2-(5-amino-3-(arylamino)-1,2,4-triazin-6-yl)-4-nitrophenyl)-2,2,2trifluoroacetamides which reacted with $N$-phenylthiourea. The structures of products were deduced from their elemental analysis and spectral measurements. The new lamotrigine analogs were evaluated in vitro as antibacterial. Interestingly, some compounds showed interesting activity against the Bacillus subtilis, Streptococcus faecalis, Micrococcus luteus, and Staphylococcus aureus bacteria.
\end{abstract}

Keywords: Fluoro/nitroaryl; Lamotrigine analogs; Thiourea; Antibacterial activity; 1,2,4-Triazines; Arylamination.

\section{Introduction}

In recent years, there has been increasing interest in the design of new drugs as to inhibition of the resistance of microbial towards the drugs were used. Among these drugs, Lamotrigine drug (3,5-diamino6-(2',4-dichlorophenyl)-1,2,4-triazine) (Fig. 1) this is used as antiepileptic, bipolar disorder 1-4, anticonvulsant, neurological lesions and act as a tranquilizer as well as behaves an effective mood stabilizer ${ }^{5,6}$. Also, Lamotrigine and its ammonium salt complexes used as antimicrobial activity ${ }^{7}$.<smiles>Nc1nnc(-c2cccc(Cl)c2Cl)c(N)n1</smiles>

Figure 1. Lamotrigine drug

Recently, Makki et al. ${ }^{8,9}$ reported a simple route to synthesize Lamotrigine analogs as an antiinflammatory and antioxidant agent. 1,2,4-triazine nucleus is scaffold of various biological activities, also have a significant variety of pharmaceutical properties, such as antitumor ${ }^{10}$, anti-HIV, antimicrobial $^{11}$. Also, isoxazole, 1,2,4-triazole,

*Corresponding author: Abdulrahman S. Alharbi

Email address: aalharbi2017@hotmail.com

DOI: http://dx.doi.org/10.13171/mjc861907296asa and benzo $[d]$ imidazole cores have a significant role in towads bacteria ${ }^{12-14}$.

Moreover, thiourea moieties play a role of biological activity involving antibacterial ${ }^{15}$, antifungal ${ }^{15,16}$, antitubercular ${ }^{17}$, antithyroid ${ }^{18}$, and insecticidal agents ${ }^{19}$.

Therefore, there is a need to develop an improved process for producing lamotrigine analogs that reduce the manufacturing cost and batch cycle time ${ }^{20}$. Thus, the present work describes a short route to obtain some new lamotrigine analogs as fluorine, arylamines, and $N$-phenylthioureas substituents given their antibacterial activity.

\section{Results and Discussion}

In the present work in the search for new highly bioactive drugs, fluorine substituted 3,5-disubstituted amino-1,2,4-triazines 11-14 as a lamotrigine analogs have been synthesized and evaluated as antibacterial probes. The starting material 2,2,2trifluoro- $N$-[2-(5-hydroxy-3-thioxo-2,3-dihydro1,2,4-triazin-6-yl)-4-nitrophenyl] aceta- mide 2 obtained from refluxing of 6-(2-amino-5nitrophenyl)-3-thioxo-3,4-dihydro-1,2,4-triazin-5 (2 H)-one 1 with ethyl 2,2,2-trifluoroacetate in THF. Compound 1 also preparing ${ }^{21}$ by refluxing 5-nitroisatin with thiosemicarbazide in $a q . \mathrm{NaOH}$ Scheme 1.

A simple primary arylamines such as 3,4-dimethylisoxazol-5-amine, 4H-1,2,4-triazol-3- 
amine, $1 H$-benzo[d]imidazol-2-amine, and 4-amino3-hydroxynaphthalene-1-sulfonic acid towards 2,2, 2 trifluoro- $N$-[2-(5-hydroxy-3-thioxo-2,3-dihydro1,2,4-triazin-6-yl)-4-nitrophenyl]acetamide $\mathbf{2}$ in refluxing isopropyl alcohol led to the formation 3-(substituted-amino)-5-hydroxy-6-aryl1,2,4-triazines 3-6 Scheme 2.

On the other hand, aminolysis of compounds 3-6 by refluxing with $\mathrm{NH}_{3} /$ EtOH afforded the 5-amino-3- (substituted-amino)-6-aryl-1,2,4-triazines $\quad \mathbf{7 - 1 0}$ as lamotrigine analogs Scheme 3.

Finally, reaction of compounds 7-10 with $\mathrm{N}$ - phenylthiourea in DMF furnished 2,2,2-trifluoro$\mathrm{N}$-[2-(3-(substituted-amino)-5-(3-phenylthioureido)1,2,4-triazin-6-yl)-4-nitrophenyl] acetamides 11-14 as lamotrigine analogs Scheme 4. Formation of compounds 11-14 may be as shown in (Fig. 2).<smiles>O=C1Nc2ccc([N+](=O)[O-])cc2C1=O</smiles>

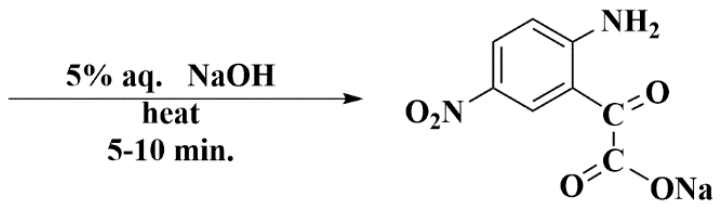<smiles>NNC(N)=S</smiles><smiles>O=C(Nc1ccc([N+](=O)[O-])cc1-c1n[nH]c(=S)nc1O)C(F)(F)F</smiles><smiles>CCOC(=O)C(C)(C)[18OH]</smiles><smiles>Nc1ccc([N+](=O)[O-])cc1-c1n[nH]c(=S)[nH]c1=O</smiles>

Scheme 1. Synthesis of compounds $\mathbf{1}$ and $\mathbf{2}$<smiles>Cc1noc(Nc2nnc(-c3cc([N+](=O)[O-])ccc3NC(=O)C(F)(F)F)c(O)n2)c1C</smiles>

3

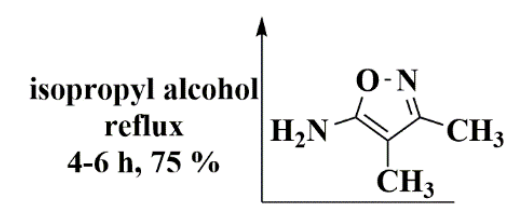<smiles>O=C(Nc1ccc([N+](=O)[O-])cc1-c1nnc(Nc2nnc[nH]2)nc1O)C(F)(F)F</smiles>

4

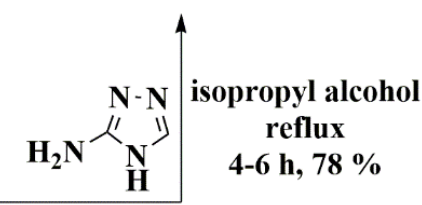

reflux

4-6 h, $78 \%$

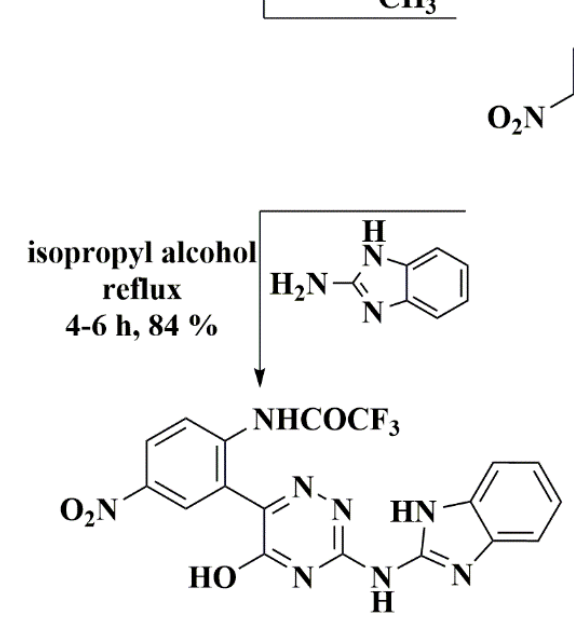

5<smiles>O=C(Nc1ccc([N+](=O)[O-])cc1-c1n[nH]c(=S)[nH]c1=O)C(F)(F)F</smiles><smiles>Nc1c(O)cc(S(=O)(=O)O)c2ccccc12</smiles><smiles>O=C(Nc1ccc([N+](=O)[O-])cc1-c1nnc(Nc2c(O)cc(S(=O)(=O)O)c3ccccc23)nc1O)C(F)(F)F</smiles>

Scheme 2. Synthesis of compounds 3-6<smiles>O=C(Nc1ccc([N+](=O)[O-])cc1-c1nnc(N[Al])nc1O)C(F)(F)F</smiles><smiles>CCC(N)(N)C(=O)O</smiles><smiles>Nc1nc(N[Al])nnc1-c1cc([N+](=O)[O-])ccc1NC(=O)C(F)(F)F</smiles> 
Scheme 3. Synthesis of compounds 7-10<smiles>CC(=O)Nc1ccc([N+](=O)[O-])cc1-c1nnc(N[17F])nc1N</smiles>

7-10

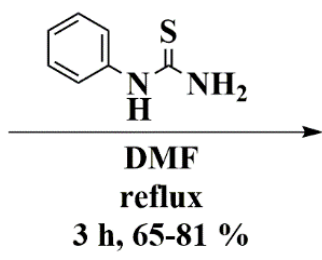

$3 \mathrm{~h}, 65-81 \%$<smiles>CC(=O)Nc1ccc([N+](=O)[O-])cc1-c1nnc(N[Al+])nc1NC(=S)Nc1ccccc1</smiles>

11-14<smiles>Cc1noc(C=[Al][Al])c1C</smiles>
$, 10,14=$<smiles>O=S(=O)(O)c1cc(O)c(I)c2ccccc12</smiles>

Scheme 4. Synthesis of compounds 11-14

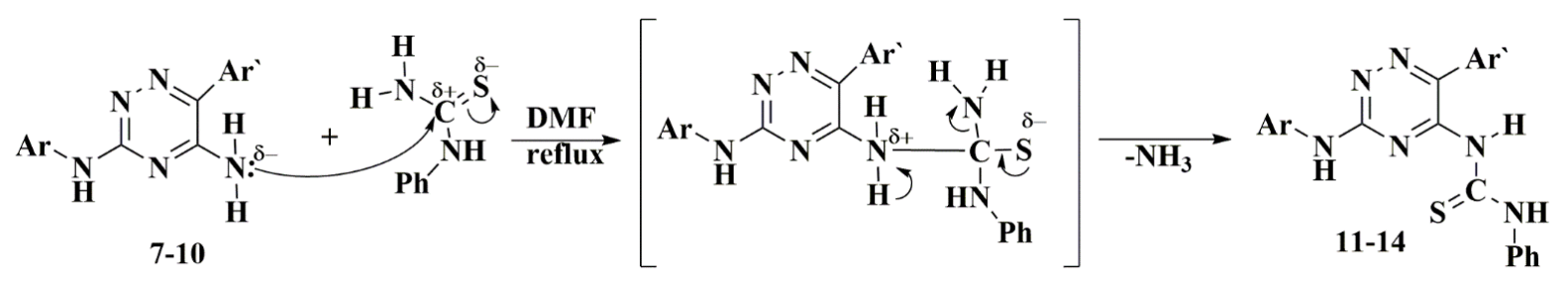

Figure 2. Formation of compounds 11-14

Structure of the new fluorinated Lamotrigine analogs deduced from their correct elemental analysis and spectral data. FT-IR spectra of the compounds $\mathbf{3}$, 7, and 11 showed $\bar{v} 2930 \sim 2905$, and $2885 \sim 2883 \mathrm{~cm}^{-}$ ${ }^{1}$ for $\mathrm{CH}_{3}$ of isoxazole moiety. Besides, ${ }^{1} \mathrm{H}$ NMR of $\mathbf{3}$, 7, and 11 exhibited two signals at $\delta 2.29 \sim 1.98$, and $2.16 \sim 1.97 \mathrm{ppm}$ for the two $\mathrm{CH}_{3}$ of isoxazole moiety.

Interestingly, the ${ }^{13} \mathrm{C} \mathrm{NMR}$ of $\mathbf{3}, \mathbf{7}$, and $\mathbf{1 1}$ showed $\delta$ at $28.8 \sim 27.0$ and $11.9 \sim 10.07 \mathrm{ppm}$ for two $\mathrm{CH}_{3} .{ }^{1} \mathrm{H}$ NMR of compounds $\mathbf{4 , ~ 8}$, and 12 showed $\delta 14.30$ $\sim 14.35 \mathrm{ppm}$ attribute to the $\mathrm{NH}$ of 1,2,4-triazole. Similarly, ${ }^{1} \mathrm{H}$ NMR of compounds 5, 9, and $\mathbf{1 3}$ showed $\delta 14.39 \sim 14.38 \mathrm{ppm}$ attribute to the NH of benzimidazole.

${ }^{1} \mathrm{H}$ NMR for compounds 1-14 exhibited $\delta$ at $13.60 \sim 13.50 \mathrm{ppm}$ referring to $\mathrm{NHCOCF}_{3}$ beside $\delta$ at $8.95 \sim 6.70 \mathrm{ppm}$ for aromatic protons. FT-IR spectra indicated the presence of $\mathrm{C}=\mathrm{O}$ at $\bar{v} 1709 \sim 1696 \mathrm{~cm}^{-1}$ and $1553 \sim 1513,1387 \sim 1370 \mathrm{~cm}^{-1}$ for asymmetrical and symmetrical for $\mathrm{NO}_{2}$ respectively for compounds 1-14.

Furthermore, FT-IR spectra of the compounds 3-6 showed a new exo-NH at $\bar{v} 3350 \sim 3190 \mathrm{~cm}^{-1}$.

Moreover, the ${ }^{1} \mathrm{H}$ NMR spectrum of 3-6 exhibited $\delta$ at $8.59 \sim 8.33 \mathrm{ppm}$ for exo $\mathrm{NH} .{ }^{13} \mathrm{C} \mathrm{NMR}$ of compounds 3-6 indicated that disappeared $\mathrm{C}=\mathrm{S}$, all of these shreds of evidence confirmed that structures of 3-6, and the arylamination of compound $\mathbf{2}$ occurred.

Structures of lamotrigine analogs 7-10 established from the presence of the vibration bands at $3441 \sim 3412$ and $3342 \sim 3300 \mathrm{~cm}^{-1}$ (stretching of $\mathrm{NH}_{2}$ ) with lacks of $\mathrm{OH}$ group. Also, ${ }^{1} \mathrm{H}$ NMR of 7-10 showed resonated signals at $\delta 4.14-4.13 \mathrm{ppm}$ attribute to $\mathrm{NH}_{2}$. Exhibition of signals at $\delta 178 \sim 176 \mathrm{ppm}$ in ${ }^{13} \mathrm{C}$ NMR of compounds 11-14 indicated that the presence of $\mathrm{C}=\mathrm{S}$ of thiourea and confirmed the reaction of compounds 7-10 with $N$-phenylthiourea 
happened. ${ }^{19} \mathrm{~F}$ NMR spectra of all compounds 1-14 showed $\delta-78 \sim-75 \mathrm{ppm}$ attribute to $\mathrm{CF}_{3}$.

Finally, the newly synthesized lamotrigine analogs (7-14) were screened for their in vitro antibacterial activity against four bacterial isolated Bacillus subtilis, Streptococcus faecalis, Micrococcus luteus, and Staphylococcus aureus. The results of the study revealed that the compounds 7-14 showed significant antibacterial potency.
As expected, compounds $\mathbf{1 2}$ and $\mathbf{1 3}$ were showed excellent activity against $B$. subtilis and $S$. faecalis bacteria, due to the presence of 1,2,4-triazine, $\mathrm{CF}_{3}$, thiourea, and 1,2,4-triazole and/ or benzo[ $d]$ imidazole moieties. On the other hand, 3-hydroxynaphthalene moiety in compounds $\mathbf{1 0}$ and $\mathbf{1 4}$ may be reduced their biological activity against the tested bacteria, because of these compounds had the lowest activities among the other synthesized compounds. The result is shown in Table 1 .

Table 1. In vitro antibacterial activity.

\begin{tabular}{|c|c|c|c|c|c|}
\hline \multirow{2}{*}{$\begin{array}{c}\text { Compound } \\
\text { No. }\end{array}$} & $\begin{array}{c}\text { Concenteration } \\
(\mu \mathrm{g} / \mathrm{mL})\end{array}$ & \multicolumn{4}{|c|}{ Inhibition zone of Bacteria (mm) } \\
\cline { 3 - 6 } & & B. subtilis & S. faecalis & M. luteus & S. aureus \\
\hline $\mathbf{7}$ & 5 & 9 & 11 & 8 & 14 \\
\hline $\mathbf{8}$ & 5 & 12 & 15 & 10 & 18 \\
\hline $\mathbf{9}$ & 5 & 10 & 13 & 12 & 15 \\
\hline $\mathbf{1 0}$ & 5 & 8 & 9 & 14 & 13 \\
\hline $\mathbf{1 1}$ & 5 & 8 & 9 & 10 & 7 \\
\hline $\mathbf{1 2}$ & 5 & 19 & 18 & 17 & 20 \\
\hline $\mathbf{1 3}$ & 5 & 18 & 21 & 20 & 9 \\
\hline $\mathbf{1 4}$ & 5 & 7 & 8 & 11 & 40 \\
\hline Ampicillin & $200 \mu \mathrm{g} / \mathrm{mL}$ & 22 & 17 & 45 & \\
\hline
\end{tabular}

\section{Conclusion}

5-Amino-3-(substituted-amino)-6-(fluoro/nitro) aryl-1,2,4-triazine and their derivatives were synthesized and evaluated against four isolated bacteria (B. subtilis, S. faecalis, M. luteus, and $S$. aureus). Some compounds exhibited a good activity towards the tested bacteria.

\section{Experimental}

All chemicals purchased from Merck and Fluka and used as received without any further purification. The melting points recorded by Stuart scientific SMP30 (Bibby, UK) melting point apparatus and reported as uncorrected. A Perkin Elmer model RXI-FT-IR 55,529 $\mathrm{cm}^{-1}$ used for recording the IR spectra. A Brucker advance DPX $400 \mathrm{MHz}$ using TMS as an internal standard used for recording the ${ }^{1} \mathrm{H},{ }^{13} \mathrm{C}$, and ${ }^{19} \mathrm{~F}$ NMR spectra at $(400 \mathrm{MHz}),(100 \mathrm{MHz})$, and $(84.25 \mathrm{MHz})$ respectively in $\mathrm{DMSO}^{-\mathrm{d}_{6}}(\delta$ in $\mathrm{ppm})$ as a solvent. Elemental microanalysis was performed on a PerkinElmer CHN-2400 analyzer. TLC analyses were performed on Merck silica gel $60 \mathrm{~F}_{254}$ aluminum plates with hexane/ethyl acetate mixtures. Compounds 1 and $\mathbf{2}$ were obtained according to the reported method ${ }^{9}$.

Synthesis of 3-(Substituted-amino)-5-hydroxy-6aryl-1,2,4-triazines 3-6

In a round-bottom flask, an equimolar amount of compound $\mathbf{2}$ and 3,4-dimethylisoxazol-5-amine, 4H-1,2,4-triazol-3-amine, $1 H$-benzo[ $d]$ imidazol-2Amine, and 4-amino-3-hydroxynaphthalene-1sulfonic acid were dissolved in isopropyl alcohol (70 $\mathrm{mL}$ ) then heated under reflux for $6 \mathrm{~h}$. The progress of the reactions was monitored by TLC. After completion of the reactions, colled at room temperature. The resulting solid was filtered off in a Buchner funnel, then washed with small amounts of cooled water and dried. Finally, the products crystallized from suitable solvents to give compounds 3-6.

\section{N-[2-(3-((3,4-Dimethylisoxazol-5-yl)amino)-5- hydroxy-1,2,4-triazin-6-yl)-4-nitrophenyl]-2,2,2- trifluoroacetamide 3:}

Orange crystals (EtOH), yield 3.621g, 75\%, M.p: 217-219 ${ }^{\circ} \mathrm{C}$.

FT-IR (ATR, $\left.\bar{v}, \mathrm{~cm}^{-1}\right)$ : 3430(OH), $3190(\mathrm{NH}), 2930$, 2885 $\left(\mathrm{CH}_{3}\right), 1697(\mathrm{C}=\mathrm{O}), 1629(\mathrm{C}=\mathrm{C}), 1520,1383$ (asym., sym. $\mathrm{NO}_{2}$ ), 1263.97(C-F).

${ }^{1} \mathrm{H}$ NMR (400 MHz, DMSO-d 6 ) $\delta$ (ppm): 13.56 $\left(\mathrm{s}, 1 \mathrm{H}, \mathrm{NHCOCF}_{3}\right), 13.16(\mathrm{~s}, 1 \mathrm{H}, \mathrm{OH}), 8.59(\mathrm{~s}, 1 \mathrm{H}$, $\mathrm{NH}), 8.31(\mathrm{~s}, 1 \mathrm{H}$, aromatic proton), 6.98, 6.76(d,d, $2 \mathrm{H}$, aromatic protons), 1.98, $1.97\left(\mathrm{~s}, \mathrm{~s}, 6 \mathrm{H}, 2 \mathrm{CH}_{3}\right)$.

${ }^{13} \mathrm{C} \quad \mathrm{NMR} \quad\left(100 \mathrm{MHz}, \quad \mathrm{DMSO}-\mathrm{d}_{6}\right) \quad \delta \quad(\mathrm{ppm}):$ 173.46(C=O), 153.59(C=N), 145.98(C-F), 135.11 $(\mathrm{C}=\mathrm{N}), \quad 128.03-126.48$ (aromatic carbons), 114.42, 113.79(C5, C6 of 1,2,4-triazine), 27, $11.9\left(2 \mathrm{CH}_{3}\right)$.

${ }^{19} \mathrm{~F}$ NMR (84.25 MHz, DMSO-d 6$) \quad \delta \quad$ (ppm): $-78 \sim-75\left(J_{\mathrm{C}-\mathrm{F}}=259 \mathrm{~Hz}, \mathrm{CF}_{3}\right)$.

Calculated: $\mathrm{C}_{16} \mathrm{H}_{12} \mathrm{~F}_{3} \mathrm{~N}_{7} \mathrm{O}_{5} \quad\left(\mathrm{M}^{+}\right.$439): C, 43.74; $\mathrm{H}, 2.75$; N, 22.32\%. Found: C, 43.52; H, 2.68; $\mathrm{N}, 22.07 \%$.

\section{$\mathrm{N}$-[2-(3-((4H-1,2,4-Triazol-3-yl)amino)-5-hydroxy- 1,2,4-triazin-6-yl)-4-nitrophenyl]-2,2,2-trifluoro- acetamide 4:}

Orange crystals (EtOH), yield 3.526g, 78\%, M.p: $254-256^{\circ} \mathrm{C}$. 
FT-IR (ATR, $\left.\bar{v}, \quad \mathrm{~cm}^{-1}\right): \quad 3415(\mathrm{OH}), \quad 3320(\mathrm{NH})$, 1696(C=O), 1616(C=C), 1522, 1377 (asym., sym. $\left.\mathrm{NO}_{2}\right), 1239(\mathrm{C}-\mathrm{F})$.

${ }^{1} \mathrm{H}$ NMR (400 MHz, DMSO-d 6 ) $\delta$ (ppm): 14.30 $\left(\mathrm{s}, 1 \mathrm{H}, \mathrm{NH}\right.$-triazole), $13.56\left(\mathrm{~s}, 1 \mathrm{H}, \mathrm{NHCOCF}_{3}\right)$, 13.17(s, $1 \mathrm{H}, \mathrm{OH}), 8.58(\mathrm{~s}, 1 \mathrm{H}, \mathrm{NH}), 8.32(\mathrm{~s}, 1 \mathrm{H}$, aromatic proton), $6.99,6.76(\mathrm{~d}, \mathrm{~d}, 2 \mathrm{H}$, aromatic proton), 6.55(s, 1H, C5- triazole). ${ }^{13} \mathrm{C}$ NMR (100 MHz, DMSO-d $\left.\mathrm{d}_{6}\right) \delta(\mathrm{ppm}): 173.41(\mathrm{C}=\mathrm{O}), 153.59$, $152.75(\mathrm{C}=\mathrm{N}), 145.89(\mathrm{C}-\mathrm{F}), 135.11(\mathrm{C}=\mathrm{N}), 128.03$ 126.48 (aromatic carbons) 114.42, 113.79(C5, C6 of 1,2,4-triazine).

${ }^{19} \mathrm{~F}$ NMR (84.25 MHz, DMSO-d 6 ) $\delta$ (ppm): -78 -75 $\left(J_{\mathrm{C}-\mathrm{F}}=259 \mathrm{~Hz}, \mathrm{CF}_{3}\right)$.

Calculated: $\mathrm{C}_{13} \mathrm{H}_{8} \mathrm{~F}_{3} \mathrm{~N}_{9} \mathrm{O}_{4} \quad\left(\mathrm{M}^{+}\right.$411): $\mathrm{C}, \quad 37.97$; $\mathrm{H}, 1.96$; N, 30.65\%. Found: C, 37.75; H, 1.81; $\mathrm{N}, 30.53 \%$.

\section{N-[2-(3-((1H-Benzo[d]imidazol-2-yl)amino)-5- hydroxy-1,2,4-triazin-6-yl)-4-nitrophenyl]-2,2,2- trifluoroacetamide 5:}

Brown crystals (EtOH), yield 3.797g, 84\%, M.p: 210$212^{\circ} \mathrm{C}$.

FT-IR (ATR, $\left.\bar{v}, \quad \mathrm{~cm}^{-1}\right): \quad 3442(\mathrm{OH}), \quad 3350(\mathrm{NH})$, 1699(C=O), 1616(C=C), 1513, 1338 (asym., sym. $\left.\mathrm{NO}_{2}\right), 1258.74(\mathrm{C}-\mathrm{F})$.

${ }^{1} \mathrm{H}$ NMR (400 MHz, DMSO-d 6 ) $\delta$ (ppm): 14.39 (s, 1H, NH-benzoimidazole), 13.54(s, $1 \mathrm{H}$, $\left.\mathrm{NHCOCF}_{3}\right), 12.50(\mathrm{~s}, 1 \mathrm{H}, \mathrm{OH}), 8.33(\mathrm{~s}, 1 \mathrm{H}, \mathrm{NH}), 8.94-$ 6.74( $\mathrm{m}, 8 \mathrm{H}$, aromatic proton).

${ }^{13} \mathrm{C} \quad \mathrm{NMR} \quad\left(100 \mathrm{MHz}, \mathrm{DMSO}-\mathrm{d}_{6}\right) \quad \delta \quad(\mathrm{ppm})$ : 173.41 $(\mathrm{C}=\mathrm{O}), \quad 168.13-152.75(\mathrm{C}=\mathrm{N}), \quad 145.89(\mathrm{C}-\mathrm{F})$, 135.12 $(\mathrm{C}=\mathrm{N}), \quad 128.03-121.35$ (aromatic carbons) 118.51, 109.44(C5, C6 of 1,2,4-triazine).

${ }^{19} \mathrm{~F}$ NMR (84.25 MHz, DMSO-d 6 ) $\delta(\mathrm{ppm})$ :

$78 \sim-75\left(J_{\mathrm{C}-\mathrm{F}}=259 \mathrm{~Hz}, \mathrm{CF}_{3}\right)$.

Calculated: $\mathrm{C}_{18} \mathrm{H}_{11} \mathrm{~F}_{3} \mathrm{~N}_{8} \mathrm{O}_{4} \quad\left(\mathrm{M}^{+} 460\right): \quad \mathrm{C}, \quad 46.97$; $\mathrm{H}, 2.41 ; \mathrm{N}, 24.34 \%$. Found: C, 46.84; H, 2.36; $\mathrm{N}, 24.19 \%$.

\section{3-Hydroxy-4-((5-hydroxy-6-(5-nitro-2-(2,2,2- trifluoroacetamido)phenyl)-1,2,4-triazin-3-yl) amino)naphthalene-1-sulfonic acid 6 :}

Black crystals (EtOH), yield 5.354g, 86\%, M.p: $248-250^{\circ} \mathrm{C}$.

FT-IR (ATR, $\left.\bar{v}, \mathrm{~cm}^{-1}\right): 3446(\mathrm{OH}), 3300(\mathrm{OH})$, 3210 $(\mathrm{NH}), \quad 1699(\mathrm{C}=\mathrm{O}), \quad 1613(\mathrm{C}=\mathrm{C}), \quad 1553$, 1370(asym., sym. $\mathrm{NO}_{2}$ ), 1237(C-F).

${ }^{1} \mathrm{H}$ NMR (400 MHz, DMSO-d $\left.{ }_{6}\right) \delta(\mathrm{ppm}): 13.60$ $\left(\mathrm{s}, 1 \mathrm{H}, \mathrm{NHCOCF}_{3}\right), 12.45(\mathrm{~s}, 1 \mathrm{H}, \mathrm{OH}), 8.60,8.59$ $(\mathrm{s}, \mathrm{s}, \quad 2 \mathrm{H}, \mathrm{OH}), \quad 8.33(\mathrm{~s}, \quad 1 \mathrm{H}, \mathrm{NH}), \quad 8.95-8.00$, 7.99-6.74(m, 9H, aromatic proton).

${ }^{13} \mathrm{C} \quad \mathrm{NMR} \quad\left(100 \mathrm{MHz}, \quad \mathrm{DMSO}-\mathrm{d}_{6}\right) \quad \delta \quad(\mathrm{ppm})$ : 173.44(C=O), 167.51-152.80 $(\mathrm{C}=\mathrm{N}), \quad 145.85(\mathrm{C}-\mathrm{F})$, 138.86, $135.11(\mathrm{C}=\mathrm{N}), \quad 128.03-121.28$ (aromatic carbons), 114.44, 112.39(C5, C6 of 1,2,4-triazine).

${ }^{19} \mathrm{~F}$ NMR (84.25 MHz, DMSO-d 6 ) $\delta$ (ppm): $-78 \sim-75$ $\left(J_{\mathrm{C}-\mathrm{F}}=259 \mathrm{~Hz}, \mathrm{CF}_{3}\right)$.

Calculated: $\mathrm{C}_{21} \mathrm{H}_{13} \mathrm{~F}_{3} \mathrm{~N}_{6} \mathrm{O}_{8} \mathrm{~S} \quad\left(\mathrm{M}^{+} 566\right): \mathrm{C}, 44.53$; $\mathrm{H}, 2.31$; N, 14.84\%. Found: C, 44.14; H, 2.23; $\mathrm{N}, 14.79 \%$.
Synthesis of 5-Amino-3-(substituted-amino)-6-aryl1,2,4-triazines 7-10

In a round-bottom flask, a mixture of compounds 3-6 $(6.5 \mathrm{mmol})$ and ammonia $(37 \%, 40 \mathrm{ml})$ in ethanol $(50 \mathrm{ml})$ heated under reflux for 4-6 h. Progress of the reactions was monitored by TLC. After completion of the reactions, cooled at room temperature then poured onto ice-drops $\mathrm{AcOH}$. The yielded solids filtered off in a Buchner funnel, then washed with a small amount of cooled water, dried. Finally, the products crystallized from proper solvents, to give compounds 7-10 respectively.

N-(2-(5-Amino-3-((3,4-dimethylisoxazol-5-yl) amino)-1,2,4-triazin-6-yl)-4-nitrophenyl)-2,2,2trifluoroacetamide 7:

Black crystals (THF), yield 2.047g, 72\%, M.p: 224-226 ${ }^{\circ} \mathrm{C}$

FT-IR (ATR, $\left.\bar{v}, \mathrm{~cm}^{-1}\right): 3412.5,3300\left(\mathrm{NH}_{2}\right)$, 3197(NH), 3152(NH), 2909, 2883.7 (aliphatic $\mathrm{CH}_{3}$ ), 1699.75(C=O), 1614.9(C=C), 1519, 1470(asym., sym. $\left.\mathrm{NO}_{2}\right), 1268.7(\mathrm{C}-\mathrm{F})$.

${ }^{1} \mathrm{H}$ NMR (400 MHz, DMSO-d 6 ) $\delta$ (ppm): 13.57 $\left(\mathrm{s}, 1 \mathrm{H}, \mathrm{NHCOCF}_{3}\right), 8.58(\mathrm{~s}, 1 \mathrm{H}, \mathrm{NH}), 8.33(\mathrm{~s}, 1 \mathrm{H}$, aromatic proton), 6.93, 6.74(d, d, $2 \mathrm{H}$, aromatic proton), 4.14(s, $\left.2 \mathrm{H}, \mathrm{NH}_{2}\right), 2.29,2.16\left(\mathrm{~s}, \mathrm{~s}, 6 \mathrm{H}, 2 \mathrm{CH}_{3}\right)$.

${ }^{13} \mathrm{C}$ NMR (100 MHz, DMSO-d 6 ) $\delta(\mathrm{ppm})$ :

173.40 $(\mathrm{C}=\mathrm{O}), 162.9(\mathrm{C}=\mathrm{N}), 145.88(\mathrm{C}-\mathrm{F})$,

135.11 $(\mathrm{C}=\mathrm{N}), 128.03-126.48$ (aromatic carbons),

116.09, 113.69(C5, C6 of 1,2,4-triazine), 28.8,

$10.73\left(2 \mathrm{CH}_{3}\right)$.

${ }^{19} \mathrm{~F}$ NMR (84.25 MHz, DMSO-d 6 ) $\delta$ (ppm): -78 -75 $\left(J_{\mathrm{C}-\mathrm{F}}=259 \mathrm{~Hz}, \mathrm{CF}_{3}\right)$.

Calculated: $\mathrm{C}_{16} \mathrm{H}_{13} \mathrm{~F}_{3} \mathrm{~N}_{8} \mathrm{O}_{4}\left(\mathrm{M}^{+} 438\right)$ : $\mathrm{C}, 43.84$;

H, 2.99; N, 25.56\%. Found: C, 43.81; H, 2.90; $\mathrm{N}, 25.37 \%$.

\section{$\mathrm{N}$-[2-(3-((4H-1,2,4-Triazol-3-yl)amino)-5-amino- 1,2,4-triazin-6-yl)-4-nitrophenyl]-2,2,2-trifluoro- acetamide 8:}

Black crystals (THF), yield 1.812g, 68\%, M.p: $251-253^{\circ} \mathrm{C}$

FT-IR (ATR, $\left.\bar{v}, \mathrm{~cm}^{-1}\right)$ : 3425, $3325\left(\mathrm{NH}_{2}\right), 3197(\mathrm{NH})$, 3150(NH), 1699.92(C=O), 1614.86(C=C), 1522, 1481 (asym., sym. $\mathrm{NO}_{2}$ ), 1267.4(C-F).

${ }^{1} \mathrm{H}$ NMR (400 MHz, DMSO-d $\left.{ }_{6}\right) \delta$ (ppm): 14.35 (s, 1H, NH-triazole), 13.57(s, 1H, $\left.\mathrm{NHCOCF}_{3}\right), 8.32(\mathrm{~s}$, $1 \mathrm{H}, \mathrm{NH}), 8.00(\mathrm{~s}, 1 \mathrm{H}$, aromatic proton $), 7.08,6.76(\mathrm{~d}, \mathrm{~d}$, $2 \mathrm{H}$, aromatic proton), 6.74(s, $1 \mathrm{H}, \mathrm{C} 5-$ triazole). 4.13(s, 2H, $\left.\mathrm{NH}_{2}\right) .{ }^{13} \mathrm{C}$ NMR (100 MHz, DMSO-d 6 ) $\delta(\mathrm{ppm}): \quad 173.41(\mathrm{C}=\mathrm{O}), \quad 153.59, \quad 152.75(\mathrm{C}=\mathrm{N})$, 145.88(C-F), 135.12(C=N), 128.03-126.48 (aromatic carbons) $114.44,113.70$ (C5, C6 of 1,2,4-triazine).

${ }^{19} \mathrm{~F}$ NMR (84.25 MHz, DMSO-d 6 ) $\delta$ (ppm): $-78 \sim-75$ $\left(J_{\mathrm{C}-\mathrm{F}}=259 \mathrm{~Hz}, \mathrm{CF}_{3}\right)$.

Calculated: $\quad \mathrm{C}_{13} \mathrm{H}_{9} \mathrm{~F}_{3} \mathrm{~N}_{10} \mathrm{O}_{3} \quad\left(\mathrm{M}^{+} 410\right)$ : C, 38.06; $\mathrm{H}, 2.21$; N, 34.14\%. Found: C, 37.87; H, 2.05; $\mathrm{N}, 34.00 \%$. 
$\mathrm{N}-[2-(3-((1 \mathrm{H}-B e n z o[d] i m i d a z o l-2-y l) a m i n o)-5-$ amino-1,2,4-triazin-6-yl)-4-nitrophenyl]-2,2,2trifluoroacetamide 9:

Black crystals (THF), yield $2.267 \mathrm{~g}, 76 \%$, M.p: 242-244 ${ }^{\circ} \mathrm{C}$.

FT-IR (ATR, $\left.\bar{v}, \mathrm{~cm}^{-1}\right): 3441,3329\left(\mathrm{NH}_{2}\right), 3195.7(\mathrm{NH})$, $3165(\mathrm{NH}), \quad 1699.7(\mathrm{C}=\mathrm{O}), \quad 1614.74(\mathrm{C}=\mathrm{C}), \quad 1521$, 1481(asym., sym. $\mathrm{NO}_{2}$ ), $1266.77(\mathrm{C}-\mathrm{F})$.

${ }^{1} \mathrm{H}$ NMR (400 MHz, DMSO-d 6 ) $\delta$ (ppm): 14.39 (s, 1H, NH-benzoimidazole), 13.56(s, $1 \mathrm{H}$, $\left.\mathrm{NHCOCF}_{3}\right), 8.32(\mathrm{~s}, 1 \mathrm{H}, \mathrm{NH}), 8.94-6.74(\mathrm{~m}, 8 \mathrm{H}$, aromatic proton).

${ }^{13} \mathrm{C} \quad$ NMR $\quad(100 \quad \mathrm{MHz}, \quad$ DMSO-d 6$) \quad \delta(\mathrm{ppm})$ : 173.14(C=O), $162.11-153.75(\mathrm{C}=\mathrm{N}), \quad 145.89(\mathrm{C}-\mathrm{F})$, $135.12(\mathrm{C}=\mathrm{N}), \quad 128.03-121.36$ (aromatic carbons) 121.36, 113.73(C5, C6 of 1,2,4-triazine).

${ }^{19} \mathrm{~F}$ NMR (84.25 MHz, DMSO-d 6 ) $\delta$ (ppm): $-78 \sim-75$ $\left(J_{\mathrm{C}-\mathrm{F}}=259 \mathrm{~Hz}, \mathrm{CF}_{3}\right)$.

Calculated: $\mathrm{C}_{18} \mathrm{H}_{12} \mathrm{~F}_{3} \mathrm{~N}_{9} \mathrm{O}_{3} \quad\left(\mathrm{M}^{+} 459\right): \quad \mathrm{C}, \quad 47.07$; $\mathrm{H}, 2.63$; N, 27.44\%. Found: C, 46.86; H, 2.46; $\mathrm{N}, 27.25 \%$.

4-[(5-Amino-6-(5-nitro-2-(2,2,2-trifluoroacetamido)phenyl)-1,2,4-triazin-3-yl)amino]-3-hydroxy naphthalene-1-sulfonic acid 10:

Black crystals (THF), yield 2.974g, 81\%, M.p: 230-232 ${ }^{\circ} \mathrm{C}$.

FT-IR (ATR, $\left.\bar{v}, \mathrm{~cm}^{-1}\right): 3436,3342\left(\mathrm{NH}_{2}\right), 3300(\mathrm{OH})$, $3186(\mathrm{NH}), \quad 3165(\mathrm{NH}), \quad 1699(\mathrm{C}=\mathrm{O}), \quad 1614(\mathrm{C}=\mathrm{C})$, 1521, 1380(asym., sym. $\mathrm{NO}_{2}$ ), 1254(C-F).

${ }^{1} \mathrm{H}$ NMR (400 MHz, DMSO-d 6 ) $\delta$ (ppm): 13.56 (s, $\left.1 \mathrm{H}, \mathrm{NHCOCF}_{3}\right), 8.58,8.47(\mathrm{~s}, \mathrm{~s}, 2 \mathrm{H}, \mathrm{OH}), 8.32$ (s, 1H, NH), 8.94-8.01, 7.98-6.74(m, 9H, aromatic proton), 4.13(s, $\left.2 \mathrm{H}, \mathrm{NH}_{2}\right)$.

${ }^{13} \mathrm{C} \quad$ NMR $\left(100 \mathrm{MHz}, \quad\right.$ DMSO-d $\left.\mathrm{d}_{6}\right) \quad \delta \quad(\mathrm{ppm})$ : 173.45(C=O), 164.02-152.81(C=N), 145.87(C-F), 138.86, $135.11(\mathrm{C}=\mathrm{N}), \quad 128.03-121.21 \quad$ (aromatic carbons), 114.43, 112.35(C5, C6 of 1,2,4-triazine).

${ }^{19} \mathrm{~F}$ NMR (84.25 MHz, DMSO-d 6 ) $\delta(\mathrm{ppm}):-78 \sim-75$ $\left(J_{\mathrm{C}-\mathrm{F}}=259 \mathrm{~Hz}, \mathrm{CF}_{3}\right)$.

Calculated: $\mathrm{C}_{21} \mathrm{H}_{14} \mathrm{~F}_{3} \mathrm{~N}_{7} \mathrm{O}_{7} \mathrm{~S} \quad\left(\mathrm{M}^{+} 565\right)$ : $\quad \mathrm{C}, \quad 44.61$; $\mathrm{H}, 2.50 ; \mathrm{N}, 17.34 \%$. Found: $\mathrm{C}, 44.56 ; \mathrm{H}, 2.47$; $\mathrm{N}, 17.08 \%$.

Synthesis of 2,2,2-Trifluoro-N-(2-(3-(substitutedamino)-5-(3-phenylthioureido)-1,2,4-triazin-6-yl)-4nitrophenyl)acetamides 11-14

In a round-bottom flask, equimolar amounts of compounds 7-10 and $N$-phenylthiourea in DMF (40 $\mathrm{ml}$ ) heated under reflux for $3 \mathrm{~h}$. Progress of the reactions was monitored by TLC. After completion of the reactions, colled at room temperature then poured onto ice. The resulting solids filtered off in a Buchner funnel, then washed with small amounts of cooled water, dried. Finally, the products crystallized from suitable solvents to give compounds 11-14, respectively.

N-[2-(3-((3,4-Dimethylisoxazol-5-yl)amino)-5-(3phenylthioureido)-1,2,4-triazin-6-yl)-4-nitrophenyl]-2,2,2-trifluoroacetamide 11:
Black crystals (Dioxane), yield $0.827 \mathrm{~g}, 81 \%$, M.p: $273-275^{\circ} \mathrm{C}$.

FT-IR (ATR, $\left.\bar{v}, \mathrm{~cm}^{-1}\right): 3450-3125(\mathrm{NH}, \mathrm{NH}, \mathrm{NH})$, 2905, 2884(aliphatic $\left.\quad \mathrm{CH}_{3}\right), \quad$ 1709.01 $(\mathrm{C}=\mathrm{O})$, 1625(C=C), 1523.37, 1482(asym., sym. $\mathrm{NO}_{2}$ ), $1250(\mathrm{C}-\mathrm{F})$.

${ }^{1} \mathrm{H}$ NMR (400 MHz, DMSO-d $\left.\mathrm{d}_{6}\right) \delta(\mathrm{ppm}): 13.60$ (s, $\left.1 \mathrm{H}, \mathrm{NHCOCF}_{3}\right), 9.65,9.52(\mathrm{~s}, \mathrm{~s}, 2 \mathrm{H}, \mathrm{NH}, \mathrm{NH})$, $8.69(\mathrm{~s}, 1 \mathrm{H}, \mathrm{NH}), 8.33-6.78(\mathrm{~m}, 8 \mathrm{H}$, aromatic proton), $2.29,1.98\left(\mathrm{~s}, \mathrm{~s}, 6 \mathrm{H}, 2 \mathrm{CH}_{3}\right)$.

${ }^{13} \mathrm{C} \quad$ NMR $\quad\left(100 \quad \mathrm{MHz}, \quad\right.$ DMSO-d $\left.\mathrm{d}_{6}\right) \quad \delta \quad(\mathrm{ppm}):$ 177.6(C=S), 163(C=N), 145.25(C-F), 135.42(C=N), 128-126 (aromatic carbons), 115.69, 112.45(C5, C6 of 1,2,4-triazine), 27.39, 10.78( $\left(2 \mathrm{CH}_{3}\right)$.

${ }^{19} \mathrm{~F}$ NMR (84.25 MHz, DMSO-d 6 ) $\delta$ (ppm): $-78 \sim-75$ $\left(J_{\mathrm{C}-\mathrm{F}}=259 \mathrm{~Hz}, \mathrm{CF}_{3}\right)$.

Calculated: $\mathrm{C}_{23} \mathrm{H}_{18} \mathrm{~F}_{3} \mathrm{~N}_{9} \mathrm{O}_{4} \mathrm{~S} \quad\left(\mathrm{M}^{+} 573\right): \quad \mathrm{C}, \quad 48.17$; H, 3.16; N, 21.98\%. Found: C, 47.94; H, 3.02; $\mathrm{N}, 21.74 \%$.

$\mathrm{N}$-[2-(3-((4H-1,2,4-Triazol-3-yl)amino)-5-(3-phenylthioureido)-1,2,4-triazin-6-yl)-4-nitrophenyl]-2,2 ,2-trifluoroacetamide 12:

Black crystals (Dioxane), yield 0.786g, 76\%, M.p: $265-268^{\circ} \mathrm{C}$.

FT-IR (ATR, $\left.\bar{v}, \mathrm{~cm}^{-1}\right)$ : 3447-3153(NH, NH, NH), $1702.21(\mathrm{C}=\mathrm{O}), 1615(\mathrm{C}=\mathrm{C}), 1521.79,1484$ (asym., sym. $\left.\mathrm{NO}_{2}\right), 1262.5(\mathrm{C}-\mathrm{F})$.

${ }^{1} \mathrm{H}$ NMR (400 MHz, DMSO-d $\left.\mathrm{d}_{6}\right) \delta(\mathrm{ppm}): 14.32$ (s, $1 \mathrm{H}, \quad \mathrm{NH}$-triazole), $13.58\left(\mathrm{~s}, 1 \mathrm{H}, \mathrm{NHCOCF}_{3}\right), 9.69$, 9.67(s,s, 2H, NH, NH), 8.61(s, 1H, NH), 8.73-7.08 $(\mathrm{m}, 8 \mathrm{H}$, aromatic proton), $6.70(\mathrm{~s}, 1 \mathrm{H}, \mathrm{C} 5$-triazole).

${ }^{13} \mathrm{C}$ NMR (100 MHz, DMSO-d 6 ) $\delta$ (ppm): $178(\mathrm{C}=\mathrm{S})$, $173(\mathrm{C}=\mathrm{O}), \quad 153.42, \quad 152.71(\mathrm{C}=\mathrm{N}), 145.89 \quad(\mathrm{C}-\mathrm{F})$, $135.12(\mathrm{C}=\mathrm{N}), \quad 127.93-125.27$ (aromatic carbons), 115.42, 113.29(C5, C6 of 1,2,4-triazine).

${ }^{19} \mathrm{~F}$ NMR (84.25 MHz, DMSO-d 6 ) $\delta$ (ppm): $-78 \sim-75$ $\left(J_{\mathrm{C}-\mathrm{F}}=259 \mathrm{~Hz}, \mathrm{CF}_{3}\right)$.

Calculated: $\mathrm{C}_{20} \mathrm{H}_{14} \mathrm{~F}_{3} \mathrm{~N}_{11} \mathrm{O}_{3} \mathrm{~S} \quad\left(\mathrm{M}^{+} 545\right): \quad \mathrm{C}, \quad 44.04$; H, 2.59; N, 28.25\%. Found: C, 43.89; H, 2.37; $\mathrm{N}, 28.12 \%$.

$\mathrm{N}$-[2-(3-((1H-Benzo[d]imidazol-2-yl)amino)-5-(3phenylthioureido)-1,2,4-triazin-6-yl)-4-nitrophenyl ]-2,2,2-trifluoroacetamide 13:

Black crystals (Dioxane), yield 0.733g, 65\%, M.p: $146-148^{\circ} \mathrm{C}$.

FT-IR (ATR, $\left.\bar{v}, \mathrm{~cm}^{-1}\right): 3453-3179(\mathrm{NH}, \mathrm{NH}, \mathrm{NH})$, 1709(C=O), 1624(C=C), 1523.37, 1487(asym., sym. $\left.\mathrm{NO}_{2}\right), 1262(\mathrm{C}-\mathrm{F})$.

${ }^{1} \mathrm{H}$ NMR (400 MHz, DMSO-d $\left.\mathrm{d}_{6}\right) \delta(\mathrm{ppm}): 14.38$ (s,1H,NH-benzoimidazole), 13.57(s, $1 \mathrm{H}, \mathrm{NHCOCF}_{3}$ ), 9.70, 9.69(s,s, 2H, NH, NH), $8.34(\mathrm{~s}, 1 \mathrm{H}, \mathrm{NH})$, 8.93-6.76(m, 13H, aromatic proton).

${ }^{13} \mathrm{C}$ NMR (100 MHz, DMSO-d 6 ) $\delta(\mathrm{ppm}): 177(\mathrm{C}=\mathrm{S})$, 173(C=O), 163-153(C=N), 145(C-F), 135(C=N), 127-121(aromatic carbons), 114, 112(C5, C6 of 1,2,4triazine).

${ }^{19}$ F NMR (84.25 MHz, DMSO-d 6 ) $\delta(\mathrm{ppm}):-78 \sim-75$ $\left(J_{\mathrm{C}-\mathrm{F}}=259 \mathrm{~Hz}, \mathrm{CF}_{3}\right)$. 
Calculated: $\mathrm{C}_{25} \mathrm{H}_{17} \mathrm{~F}_{3} \mathrm{~N}_{10} \mathrm{O}_{3} \mathrm{~S} \quad\left(\mathrm{M}^{+} 594\right): \quad \mathrm{C}, \quad 50.51$; $\mathrm{H}, 2.88$; N, 23.56\%. Found: C, 50.36; H, 2.69; $\mathrm{N}, 23.46 \%$.

\section{3-Hydroxy-4-[(6-(5-nitro-2-(2,2,2-trifluoroacetam- ido)phenyl)-5-(3-phenylthioureido)-1,2,4-triazin-3 - yl)amino])naphthalene-1-sulfonic acid 14:}

Black crystals (Dioxane), yield 0.944g, 71\%, M.p: $228-230^{\circ} \mathrm{C}$

FT-IR (ATR, $\left.\bar{v}, \mathrm{~cm}^{-1}\right)$ : 3530-3186.64(4NH, OH), $1707(\mathrm{C}=\mathrm{O}), 1615(\mathrm{C}=\mathrm{C}), 1522,1381$ (asym., sym. $\left.\mathrm{NO}_{2}\right), 1271(\mathrm{C}-\mathrm{F})$.

${ }^{1} \mathrm{H}$ NMR (400 MHz, DMSO-d 6 ) $\delta$ (ppm): 13.50 (s, 1H, $\left.\mathrm{NHCOCF}_{3}\right), 9.69,9.67(\mathrm{~s}, \mathrm{~s}, 2 \mathrm{H}, \mathrm{NH}, \mathrm{NH})$, $8.68, \quad 8.57(\mathrm{~s}, \mathrm{~s}, \quad 2 \mathrm{H}, \quad \mathrm{OH}), \quad 8.34(\mathrm{~s}, \quad 1 \mathrm{H}, \quad \mathrm{NH})$, $8.90-7.20$ ( $\mathrm{m}, 14 \mathrm{H}$, aromatic proton).

${ }^{13} \mathrm{C}$ NMR (100 MHz, DMSO-d 6 ) $\delta(\mathrm{ppm}): 176(\mathrm{C}=\mathrm{S})$, $173(\mathrm{C}=\mathrm{O}), \quad 163-153 \quad(\mathrm{C}=\mathrm{N}), \quad 145(\mathrm{C}-\mathrm{F}), \quad 137$, $134(\mathrm{C}=\mathrm{N}), 127-121$ (aromatic carbons), 114, 112(C5, C6 of 1,2,4-triazine).

${ }^{19} \mathrm{~F}$ NMR (84.25 MHz, DMSO-d 6 ) $\delta$ (ppm): $-78 \sim-75$ $\left(J_{\mathrm{C}-\mathrm{F}}=259 \mathrm{~Hz}, \mathrm{CF}_{3}\right)$.

Calculated: $\mathrm{C}_{28} \mathrm{H}_{19} \mathrm{~F}_{3} \mathrm{~N}_{8} \mathrm{O}_{7} \mathrm{~S}_{2} \quad\left(\mathrm{M}^{+} 700\right)$ : C, 48.00; $\mathrm{H}, 2.73$; N, 15.99\%. Found: C, 47.85; H, 2.64; $\mathrm{N}, 15.74 \%$.

\section{The antibacterial activity}

The in vitro antimicrobial activity of lamotrigine analogs 7-14 was screened against some selected bacterial (Bacillus subtilis, Streptococcus faecalis, Micrococcus luteus, and Staphylococcus aureus) by the reported method. The suspension of each microorganism rubbed onto the surface of solidified nutrient agar already set into Petri dishes with swap stick.

The stock solution suitably diluted to get dilution of $5 \mu \mathrm{g} / \mathrm{mL}$ concentration (DMSO) of the tested compounds 7-14. Wells (6 $\mathrm{mm}$ in diameter) dug in the agar media with the help of a sterile metallic borer. Ampicillin $200 \mu \mathrm{g} / \mathrm{mL}$ used as controls. The wells incubated immediately at $37^{\circ} \mathrm{C}$ for $48 \mathrm{~h}$. The activity determined by measuring the diameter of zones indicating complete inhibition ( $\mathrm{mm}$ ) and comparing the values with the standard. ${ }^{22}$

\section{Conflict of interest}

The authors declare no conflict of interest.

\section{Acknowledgements}

We would like to express our sincere gratitude to King Abdulaziz University.

\section{References}

1- J. M. Zakrzewska, Z. Chaudhry, T. J. Nurmikko, Patton, D. W., Mullens, E. L., Lamotrigine (Lamictal) in refractory trigeminal neuralgia: results from a double-blind placebo- controlled crossover trial. Pain, 1997, 73 (2), 223-230. https://doi.org/10.1016/S03043959(97)00104-8.

2- M. Rowbotham, N. Harden, B. Stacey, P. Bernstein, L. Magnus-Miller, For the Gabapentin Postherpetic Neuralgia Study, G. Gabapentin for the treatment of postherpetic neuralgia: A randomized controlled trial. JAMA, 1998, 280(21), 1837-1842. http://dx.doi .org/10.1001/jama.280.21.1837.

3- D. R. Goldsmith, A. J. Wagstaff, T. Ibbotson, C. M. Perry. Lamotrigine, Drugs, 2003, 63(19), 2029-2050. https://doi.org/10.2165/00003495200363190-00009.

4- J. R. Calabrese, E. Vieta, M. D. Shelton, Latest maintenance data on lamotrigine in bipolar disorder. Eur. Neuropsychopharm., 2003, 13, 57-66. http://dx.doi.org/10.1016/S0924-977X (03)00079-8.

5- J. G. Hardman, L. E. Limburd, A. G. Gilman, The pharmacological basis of therapeutics, $10^{\text {th }}$ ed., New York: McGraw-Hill. ISBN 0-07135469-7., 2000.

6- S. Sweetman, Martindale: The Complete Drug Reference, 35 ed., The pharmaceutical press. In: London, 2007.

7- Y. Qian, P. C. Lv, L. Shi, R. Q. Fang, Z.C. Song, H.L. Zhu, Synthesis, antimicrobial activity of lamotrigine and its ammonium derivatives. Journal of Chemical Sciences, 2009, 121(4), 463-470.

8- M.S.T. Makki, D.A. Bakhotmah, R.M. AbdelRahman, F. M. Aqlan, New route to synthesize fluorine substituted lamotrigine drug analogs as an anti-inflammatory agent. Current Organic Synthesis, 2017, 14, 1-10. http://dx.doi.org/10. 2174/1570179414666170509151123.

9- M. S. T. Makki, R. M. Abdel-Rahman, A. S. Alharbi, Synthetic approach for novel fluorine substituted $\alpha$-amino-phosphonic acids containing 1,2,4-triazin-5-one moiety as antioxidant agents. International Journal of Organic Chemistry, 2018, 8(1), 1-15. https://doi .org/10.4236/ijoc.2018.81001.

10- S. Cascioferro, B. Parrino, V. Spanò, A. Carbone, A. Montalbano, P. Barraja, P. Diana, G. Cirrincione, An overview of the recent developments of 1,2,4-triazine derivatives as anticancer compounds. European Journal of Medicinal Chemistry, 2017, 142, 328-375.

11- M. S. T. Makki, R. M. Abdel-Rahman, A. S. Alharbi, Synthetic strategies, chemical reactivities and biological activities of 3-thioxo1,2,4-triazin-5-ones and their derivatives. MiniReviews in Organic Chemistry, 2019, 16(4), 308-322.

12- S. S. Wazalwar, A. R. Banpurkar, F. Perdih, Aqueous phase synthesis, crystal structure, and biological study of isoxazole extensions of pyrazole-4-carbaldehyde derivatives. Journal of Molecular Structure, 2017, 1150, 258-267. 
13- F. Gao, T. Wang, J. Xiao, G. Huang, Antibacterial activity study of 1,2,4-triazole derivatives. European Journal of Medicinal Chemistry, 2019, 173, 274-281.

14- G. Manjunath, G. Bheemaraju, M. Mahesh, P. Venkata Ramana, Synthesis of new 5-((2(substitutedphenoxymethyl)-1H-benzo[d]imidazole-1-yl)methyl)-1,3,4-oxadiazole-2-thiol: A novel class of potential antibacterial and antifungal agents. Annales Pharmaceutiques Françaises, 2015, 73 (6), 452-460.

15- S. Saeed, N. Rashid, P. G. Jones, M. Ali, R. Hussain, Synthesis, characterization and biological evaluation of some thiourea derivatives bearing benzothiazole moiety as potential antimicrobial and anticancer agents. European Journal of Medicinal Chemistry, 2010, 45(4), 1323-1331.

16- M. Eweis, S. S. Elkholy, M. Z. Elsabee, Antifungal efficacy of chitosan and its thiourea derivatives upon the growth of some sugar-beet pathogens. International Journal of Biological Macromolecules, 2006, 38 (1), 1-8.

17- D. Sriram, P. Yogeeswari, K. Madhu, Synthesis and in vitro antitubercular activity of some 1[(4-sub)phenyl]-3-(4-\{1-[(pyridine-4-carbonyl) hydrazono] ethyl $\}$ phenyl)thiourea. Bioorganic and Medicinal Chemistry Letters, 2006, 16 (4), 876-878.
18- D. Manna, G. Roy, G. Mugesh, Antithyroid Drugs and Their Analogues: Synthesis, Structure, and Mechanism of Action. Accounts of Chemical Research, 2013, 46(11), 27062715.

19- A. Saeed, M. N. Mustafa, M. Zain-ul-Abideen, G. Shabir, M. F. Erben, U. Flörke, Current developments in chemistry, coordination, structure and biological aspects of 1(acyl/aroyl)-3-(substituted)thioureas: advances Continue. Journal of Sulfur Chemistry, 2019, 40 (3), 312-350. https://doi.org/10.1080/174159 93.2018.1551488.

20- G. Venkanna, D. Nagender, P. Venkateswarlu, K. Chandra Shekhar, G. Madhusudhan, K. Mukkanti, Process for producing 6-(2,3dichlorophenyl)-1,2,4-triazine 3,5-diamine (Lamotrigine) and identification, characterization of a new N-methyl impurity. Der Pharma Chemica, 2012, 4(1), 100-105.

21- R. M. Abdel-Rahman, Synthesis and antihuman immune virus activity of some new fluorine-containing substituted-3-thioxo-1,2,4triazin-5-ones. Farmaco., 1991, 46(2), 379-389.

22- Atta-ur-Rahman M. I. Choudhary, W. J., Thomsen, Bioassay techniques for drug development, Harwood Academic Publishers, The Netherlands, 2001, pp. 16-19. 